\title{
Nutricionista otimizando a qualidade de vida do paciente oncológico
}

\section{Nutritionist optimizing the quality of life of cancer patients}

\author{
Rhaissa Pinheiro Ferreira \\ Instituição:Universidade da Amazonia - UNAMA, Belém - PARÁ; \\ Mayla Karla de Souza Monteiro \\ Instituição:Universidade da Amazonia - UNAMA, Belém - PARÁ; \\ Camila Lorena Rodrigues Machado. \\ Instituição:Universidade da Amazonia - UNAMA, Belém - PARÁ;
}

\begin{abstract}
RESUMO
A intervenção nutricional adequada promove bem-estar, controle de sintomas e melhora da qualidade de vida dos pacientes. Logo, o nutricionista deve orientar o paciente e sua família sobre a terapia nutricional em uso, além de fornecer orientações e esclarecimentos, a fim de prolongar a sobrevida, reduzir a perda de peso e melhorar a qualidade de vida.
\end{abstract}

Palavras-chave: Nutrição enteral, Neoplasia e Terapia nutricional.

\begin{abstract}
The adequate nutritional intervention promotes well-being, control of symptoms and improvement of the quality of life of patients. Therefore, the nutritionist should guide the patient and his family about the nutritional therapy being used, in addition to providing guidance and clarification, in order to prolong survival, reduce weight loss and improve quality of life.
\end{abstract}

Keywords: Enteral nutrition, Neoplasia, Nutritional therapy.

\section{INTRODUÇÃO}

O câncer é considerado uma enfermidade multifatorial crônico que se caracteriza pelo crescimento descontrolado, rápido e invasivo de células com alteração em seu material genético. Também é definido como uma doença catabólica que consome as reservas nutricionais do paciente devido ao aumento do gasto energético pela atividade humoral presente (BRASIL, 2016; HOFF PM, 2018).

A perda de peso e a desnutrição são os distúrbios nutricionais mais frequentes em pacientes oncológicos e são associados à diminuição da resposta ao tratamento especifico e à depressão do sistema imune, acarretando em uma perda na qualidade de vida do paciente, deixando-o mais suscetível a complicações no pós-operatório, aumento morbimortalidade, no tempo de internação e no custo hospitalar.

Em virtude disso, faz-se necessário, desde o diagnóstico e durante todo o tratamento, a presença de uma equipe multidisciplinar, em especial o nutricionista para estimular uma alimentação adequada. Propõe-se que a assistência nutricional ao paciente oncológico seja individualizada e compreenda desde a avaliação nutricional, o cálculo das necessidades nutricionais até a aplicação da terapia nutricional, com o objetivo 
de prevenir ou corrigir deficiências nutricionais e minimizar a perda de peso, mediante a alimentação oral, enteral e parenteral (BRASIL, 2016; FRANCESCHINI JP, et al.,2020)

\section{OBJETIVO}

Objetivou-se revisar, descrever e analisar as produções cientificas relacionadas com a importância do nutricionista na terapia nutricional enteral e no estado nutricional de pacientes oncológicos hospitalizados e ressaltar como esta alternativa pode ajudar como essa alternativa pode ajudar os pacientes.

\section{MÉTODO}

Trata-se de uma pesquisa que foi realizada a partir de uma revisão sistemática. Tendo em vista essa perspectiva, a revisão de literatura foi pautada e fundamentada a partir da análise de artigos, dissertações e revistas científicas obtidas na base de dados SCIELO, PUBMED e LILACS. Com a utilização de descritores para a pesquisa, como: nutricionista, câncer e terapia nutricional enteral. Utilizando como recorte temporal 2018 a 2021, no idioma em português, e foram excluídos artigos que não era relacionado com o tema, ao final, procedeu-se à análise de 5 artigos.

\section{REVISÃO BIBLIOGRÁFICA}

Em um estudo mostrou, o perfil sociodemográfico dos pacientes com câncer e que realizavam terapia nutricional enteral, onde a amostra era de 96 prontuários. O resultado encontrado foi que a maioria dos pacientes enfermos no hospital eram conveniados com o SUS, na maioria do sexo masculino, idoso, baixa escolaridade e tinha algum consumo de álcool e/ou cigarro, com isso o câncer de maior frequência é o de cabeça e pescoço (SOUZA IA, et al., 2018). A terapia nutricional enteral é muito importante nesse momento, pois a ocorrência de complicações gastrointestinais e intercorrências possam ter contribuído para a inadequação entre prescrição e infusão da dieta enteral, e consequentemente para um possível comprometimento do estado nutricional (BORTOLETTO MM, et al., 2018).

Foi observado, em um estudo observacional 39\% da amostra com o estado nutricional em desnutrição, associada à diminuição da resposta aos tratamentos antineoplásicos, à redução da qualidade de vida, aos maiores riscos de infecções, ao aumento do tempo de hospitalização e da morbimortalidade (VILLARDO GP, et al., 2018). Contudo, a necessidade de uma intervenção nutricional precoce, pode fazer diferença uma vez que esses pacientes têm seu estado nutricional comprometido de forma progressiva (GUILHERME LG, et al., 2020).

Em um ensaio clínico randomizado duplo não cego, separou sua amostra em grupo denominado intervenção (GI) e grupo padrão (GP), onde o GI recebeu aconselhamento nutricional em todos os dias da 
radioterapia. Dessa forma, a perda de peso foi menor no grupo que era acompanhado por um nutricionista, mostrando o benefício do nutricionista na equipe multiprofissional (POOTZ SC, et al,. 2020).

\section{DISCUSSÃO}

Foram analisados 5 artigos, no qual 3 compõem a revista Revista Brasileira de Cancerologia e 2 da Nutrición clínica dietética hospitalaria. É observado que alguns autores afirmam a importância do nutricionista para a avaliação e acompanhamento na parte clínica oncológica. Com isso, tendo em vista as análises dos artigos e a pequena literatura relacionada com o tema trás dúvidas da melhor terapia nutricional oferecer aos pacientes com cuidados paliativos. Portanto, são necessárias novas pesquisas que abordem atuação do nutricionista, possibilitando a construção de novas propostas.

\section{CONSIDERAÇÕES FINAIS}

Portanto, demonstrar a relevância do profissional nutricionista nos cuidados paliativos, com vista na promoção a saúde do paciente, e com o contexto multidisciplinar do cuidado paliativo oncológico tem uma participação muito importante na evolução do paciente, ajudando a equipe a traçar o melhor plano terapêutico, em relação a nutrição. Nesse sentido, esse tratamento ajuda na redução da angústia e sofrimento tão presente nesses pacientes. Visto isso, são necessários novos estudos que abordem atuação do nutricionista em pacientes oncológicos. 


\section{REFERÊNCIAS}

1. BORTOLETTO MM, et al. Perfil Sociodemográfico e Nutricional de Pacientes Oncológicos em Terapia Nutricional Enteral. Revista Brasileira de Cancerologia 2018; 64(2): 141-147

2. BRASIL. Ministério da Saúde. Consenso nacional de nutrição oncológica. 2016. Disponível em: https://www.inca.gov.br/sites/ufu.sti.inca.local/files/media/document/consenso-nutricao-oncologica-volii-2-ed-2016.pdf. Acessado em: 29 de maio de 2021.

3. FRANCESCHINI JP, et al. Manuela Pacheco Nunes da. Papel do binômio anorexia e perda de peso em pacientes com câncer de pulmão em estádio IV. J. bras. pneumol. vol.46 no.4 São Paulo 2020.

4. GUILHERME LG, et al. Terapia Nutricional em pacientes oncológicos: Realidade de um hospital de referência em Pernambuco. Nutr. clín. diet. hosp. 2020; 40(1):33-39

5. HOFF, PM. Manual de condutas em pacientes oncológicos. São Paulo: Atheneu, 2018.

6. POOTZ SC, et al. Aconselhamento Nutricional em Pacientes com Câncer de Cabeça, Pescoço e Esôfago em Tratamento (Quimio) radioterápico. Revista Brasileira de Cancerologia 2020; 665(1): e-13531

7. SOUZA IA, et al. Nutrição enteral em pacientes oncológicos: diferenças entre o que é prescrito e administrado. Nutr. clín. diet. hosp. 2018; 38(2):31-38

8. VILLARDO GP, et al. Adequação Proteica versus Estado Nutricional de Pacientes Oncológicos Adultos em Unidade de Terapia Intensiva. Revista Brasileira de Cancerologia 2018; 64(4): 527-532 